\title{
Comportamientos prosociales para mejorar la convivencia escolar en las instituciones educativas de Colombia
}

\author{
Prosocial behave for school life at the Colombia educative institutions \\ Comportamentos pró-sociais para melhorar a convivência escolar em instituições de \\ ensino na Colômbia
}

ARTÍCULO DE REVISIÓN

\author{
Nidya Yohana Murillo Serna \\ lunastarmoon@hotmail.com \\ ORCID: 0000-0002-4931-6626
}

Universidad Metropolitana de Educación, Ciencia y Tecnología, Panamá

Recibido abril 2020 | Revisado mayo 2020 | Publicado en julio 2020

\section{RESUMEN}

El artículo presenta un abordaje teórico y conceptual sobre como el comportamiento prosocial ayuda a la efectiva convivencia escolar mediante la utilización por parte de los estudiantes en sus relaciones, en el ámbito educativo, el contexto social, usando herramientas como la empatía, la solidaridad y el respeto por las diferencias. Para el abordaje se utilizó un nivel monográfico dirigido a la revisión de las fuentes para recopilar las ideas de los planteamientos conceptuales de diversos autores; luego se desarrolló un nivel hermenéutico en el cual se interpretaron los planteamientos de los autores a partir de la sustentación, vigencia y alcance; de esta manera se planteó la problemática de la convivencia y las conductas prosociales presentes en los ámbitos de la educación colombiana. Así mismo, se plantea una reflexión final sobre la importancia de la educación en la prosecución de conductas prosociales no solo para el ámbito escolar sino también para ejercitarlas en los diferentes ámbitos sociales.

Palabras clave: Convivencia escolar; prosocial; empatía, respeto

\begin{abstract}
The article presents a theoretical and conceptual approach on how prosocial behavior helps effective school coexistence through the use by students in their relationships, in the educational field, the social context, using tools such as empathy, solidarity and respect for differences. For the approach, a monographic level aimed at reviewing the sources was used to collect the ideas of the conceptual approaches of various authors; Then a hermeneutical level was developed in which the authors' approaches were interpreted from the support, validity and scope; In this way, the problem of coexistence and prosocial behaviors present in the fields of Colombian education was raised. Likewise, a final reflection is proposed on the importance of education in the prosecution of prosocial behaviors not only for the school environment but also to exercise them in different social environments.
\end{abstract}

Key words: School coexistence; prosocial; empathy, respect 


\section{RESUMO}

0 artigo apresenta uma abordagem teórica e conceitual de como o comportamento prósocial auxilia na efetiva convivência escolar por meio do uso pelos alunos em suas relações, no campo educacional, no contexto social, utilizando ferramentas como empatia, solidariedade e respeito pelas diferenças. Para a abordagem, foi utilizado um nível monográfico voltado para a revisão das fontes para coletar as ideias das abordagens conceituais de vários autores; Em seguida, desenvolveu-se um nível hermenêutico em que as abordagens dos autores foram interpretadas a partir do suporte, validade e escopo; Assim, levantou-se o problema da convivência e dos comportamentos pró-sociais presentes nos campos da educação colombiana. Da mesma forma, propõe-se uma reflexão final sobre a importância da educação no julgamento de comportamentos pró-sociais não só para o ambiente escolar, mas também para exercê-los em diferentes ambientes sociais.

Palavras-chave: Convivência escolar; pró-social; empatia, respeito

\section{INTRODUCCIÓN}

En un mundo dinámico con cambios vertiginosos y cada vez más sofisticados como consecuencia, entre otros, de los fenómenos de la globalización, se desafía a la educación a asumir la responsabilidad de formar profesionales competentes $\mathrm{y}$, además, felices; hombres que hallen en la educación las distintas maneras de movilidad sociales y económicas existentes.

El reto mencionado compromete a los actores en el proceso educativo a generar y establecer, mediante la aplicación de estrategias, espacios pedagógicos que den respuestas pertinentes en la resolución de situaciones que alteran no sólo el proyecto de vida de los discentes, sino que al mismo tiempo cercenan, mediante la siembra de antivalores, la actividad e influencia de las instituciones, en este caso educativas, en un intento desesperado de invalidar su crecimiento y posicionamiento dentro de su ámbito social y comunitario.

Esta realidad incentiva la búsqueda del fortalecimiento de competencias de convivencia y prosocialidad desde la pedagogía, para la efectiva comprensión e internalización por parte de la comunidad educativa del texto y del contexto en sus diferentes momentos académicos, sociales y laborales, que permitan la transformación de la cosmovisión, y de los procesos de autogestión y autorregulación del conocimiento y los saberes aplicados.

Consecuencialmente, el nacimiento de una nueva cosmovisión debe contener la intencionalidad de retomar espacios para hacer encuentros entre el "sí mismo" y el "otro" desde una perspectiva de respeto, de compromiso, donde el verdadero sentido de convivencia se manifieste a través de comportamientos empáticos, solidarios, cordiales que permitan el desarrollo del sentido de pertenencia, una comunicación clara y efectiva que estimule la organización y la participación de la comunidad para aceptarse y aceptar, para brindarse apoyo mutuo, para afrontar las diferentes situaciones del diario vivir y generar las soluciones adecuadas.

Tal como menciona Barroso (2009, p. 347) "hay que desmontar la información con la que se ha venido operando", lo que no es más que una invitación a comenzar a enriquecer niños, adolescentes y la sociedad en general con nuevos valores, reglas básicas, actitudes y comportamientos diferentes de autoestima y convivencia social, ya que básicamente toda sociedad es la información lo que maneja.

El mencionado proceso de enriquecimiento personal inicia en la familia, como primer círculo de influencia, pero es en la escuela donde se reafirma y afianza, mediante la labor de un maestro nutritivo con presencia $\mathrm{y}$ liderazgo que, a través de sus enseñanzas y modelamiento habilite a sus discentes y a la 
sociedad a liderar sus propias vidas y a interconectarse con todos sin tener en cuenta las diferencias existentes de cualquier orden, solo así se construye una convivencia que sirva de plataforma a un estilo de vida para el crecimiento y el desarrollo del ser humano que requieren los nuevos tiempos.

\section{MÉTODO}

Este trabajo se orientó a realizar un abordaje teórico y conceptual sobre la convivencia y las conductas prosociales presentes en los ámbitos de la educación colombiana. Para el proceso de documentación según lo dispuesto por Hurtado (2010), se utilizaron los siguientes niveles: a) Nivel monográfico: En este nivel mediante la técnica de Riaceer la cual comprende la recopilación, identificación y almacenamiento, se revisaron los diferentes aspectos conceptuales planteados por los autores sobre los elementos que conforman la convivencia y las conductas prosociales, y b). Nivel hermenéutico: donde se realizó una interpretación de los aspectos conceptuales presentados en el nivel monográfico a partir del entendimiento, sustentación, alcance y vigencia de los planteamientos.

\section{DESARROLLO Y DISCUSIÓN}

En general, la educación es el proceso de formación del individuo, se basa en la transmisión de los parámetros de la cultura específica del sistema o conformación social que la enmarca. Este proceso puede darse de manera informal, como la educación que se recibe del medio y de las interrelaciones sociales, también, en unidades sociales como la familia y la iglesia; y la educación formal referida a la recibida en instituciones que trabajan gradualmente $y$ tienden a la titulación.
En este sentido, cada sistema educativo establece las normas, valores, principios y lógicas que cohesionan y dan identidad a las personas de determinada conformación cultural. Tanto los sistemas de gobierno como los organismos internacionales establecen las ideologías, vínculos y canales para sostener el sistema educativo.

Igualmente, la educación es un proceso de formación y adecuación social fundamental para la vida del ser humano en la cultura de toda sociedad. Para la Unesco (2015), la educación es una prioridad como deberderecho humano y bien público. Esta organización ayuda a los países de América latina para desarrollar sistemas educativos inclusivos y de calidad y, a la vez, fortalece sus capacidades para formular y aplicar políticas educativas.

Desde estas perspectivas, se infiere la importancia de la educación como impulsadora del cambio y del desarrollo sostenible con calidad de vida y en armonía con la naturaleza, como se determina en las directrices del mundo globalizado actual. De esta manera, se propende el desarrollo en espacios pertinentes al digno y grato acto de la formación humana sin violencia, en sana convivencia para que ofrezca felicidad y realización de los sujetos implicados en el proceso educativo.

La región de América Latina comprende veinte (20) países con sus particularidades y divergencias $\mathrm{y}$, a su vez, con semejanzas dada su similitud étnica y procesos históricos y sociales afines en lo referente a la educación, los contextos y retos tienen similitudes que resultan difíciles de superar a pesar de las intervenciones que se han hecho al respecto frente a las dificultades. Desde México hasta Chile, cada país establece su marco legal para promover la convivencia escolar y prevenir toda forma de violencia física o psicológica, 
agresiones u hostigamientos, el delito o la disrupción en el ámbito académico.

Es así como los países latinoamericanos han avanzado en aspectos como cobertura, equidad y calidad de la educación. Sin embargo, los resultados en competencias evaluadas en las diferentes pruebas del estado desde Saber, PRO y PROTT; por supuesto, en las pruebas internacionales como las de estudio de las tendencias en matemáticas y ciencias (TIMS), y las del Programa Internacional para la Evaluación de Estudiantes (PISA), las de la Oficina Regional de Educación para América Latina y el Caribe (OREALC/UNESCO- Santiago), y las del Estudio Internacional del Progreso en Comprensión Lectora PIRLS (IEA), en las cuales participa Colombia, los resultados han sido cuestionados y se trabaja en mejorarlos. Es importante resaltar que solo nueve (9) países de américa Latina participan en las pruebas internacionales, y de esos solo tres (Chile, Colombia y Perú) han empezado a arrojar avances en comparación a versiones anteriores de las pruebas.

Para el logro de la educación pertinente y con calidad, es importante el cuidado de los espacios y ambientes educativos, por cuanto ellos permiten que los procesos de enseñanza aprendizaje se den en iguales condiciones para todos los estudiantes, es decir, se den espacios libres de discriminación donde la convivencia y la educación en comportamientos prosociales permita la cooperación, espacios donde el trabajo se realice con respeto y valoración de las diferencias, lo que asegura así, la convivencia pacífica, la calidad educativa y el pleno desarrollo de los estudiantes.

De esta manera, se entiende la convivencia escolar como la acción de vivir, en el contexto escolar, en compañía de otras personas de manera pacífica y armónica. Se refiere este concepto al conjunto de relaciones que ocurren entre las personas que hacen parte de la comunidad educativa, el cual debe enfocarse más allá del logro de los objetivos educativos, en el desarrollo integral de los estudiantes.

En este sentido, expresa Mockus (2002) que la convivencia escolar resume el ideal de la vida compartida entre las personas que componen la comunidad educativa y esta se inicia en el deseo de vivir juntos de manera operativa y deseable, muy a pesar de la diversidad de orígenes. Al respecto se han dado algunas iniciativas, en Perú por ejemplo se promulgo un Decreto Supremo $\mathrm{N}^{\circ}$ 006-2012-ED del 31 de marzo de 2012, en Argentina, la Ley 26.150; en Paraguay la Resolución № 8353/12 y el Protocolo de Atención para casos de violencia escolar; Ley No 4.633/12 contra el acoso escolar en instituciones educativas. Ecuador tiene la Ley Orgánica de Educación Intercultural (LOEI) (81) y un instructivo para la implementación del programa de participación estudiantil (13260).

En Colombia, país que ha sufrido por mucho tiempo la violencia del conflicto armado que se inició desde la década de los años cincuenta en adelante (1948-1917), esta situación ha provocado que los niveles de violencia en el país aumenten significativamente, tanto dentro de las familias, como en los adolescentes y jóvenes estudiantes del diversificado, a tal punto que la escuela es uno de los espacios más afectados por este fenómeno, situación que en los últimos veinte (20) años ha generado un desplazamiento masivo de personas, entre ellos: niños, niñas y adolescentes, cuyos derechos han sido vulnerados casi en su totalidad, incluido el de la educación.

Es por esto que para que se favorezcan los procesos de enseñanza-aprendizaje $\mathrm{y}$, además, los espacios de formación que establece el currículo, el marco legal de la educación en Colombia dicta la Ley 1620 de 
convivencia escolar; donde se establecen los manuales de convivencia, los centros o laboratorios de convivencia y la función de los mediadores escolares; todos con la finalidad de favorecer la implementación de espacios de sana convivencia y prevención del maltrato.

Así mismo, desde el enfoque curricular determinado por el Ministerio de Educación Nacional de Colombia (MEN), como los Marcos Generales, Lineamientos curriculares y el sistema por competencias, establecen la formación por competencias ciudadanas y comportamientos prosociales en el desarrollo de las diferentes áreas y sus contenidos temáticos determinados desde el Ministerio de educación Nacional (MEN). En conclusión, se rescata la importancia de la educación en comportamientos prosociales desde el ámbito legal y el curricular.

Igualmente, en el Plan Internacional Unicef (2017 c. p. Romero, 2011) se reconoce la incidencia positiva que tienen las campañas para empoderar a los niños, niñas y adolescentes sobre el conocimiento y uso de sus derechos, la promoción de la educación sin miedo, sin exclusión y sin violencia física, psicológica o sexual, ajena a la intimidación, el cyberbullying, el grooming como también la violencia basada en el género y todo lo relacionado con la venta y compra de niños, pornografía y prostitución infantil.

En efecto, en el Foro Internacional sobre Prevención y Manejo de la Intimidación Escolar, en Bogotá (2007), quedó sustentada la afirmación de que la violencia se arraiga en orígenes económicos y sociales, que son producto de las dinámicas sociopolíticas y éstas a su vez, impactan los escenarios comunitarios, entre ellos el educativo. (Vélez 2012).

Aprender a convivir es un proceso que se debe integrar y cultivar diariamente en todos los escenarios de la escuela (Pérez, 2007). Asimismo, se deben favorecer ambientes de aprendizaje democráticos, donde la participación y la construcción de identidad desde la diferencia sean centrales. Las escuelas juegan un papel decisivo para cambiar los patrones de violencia y para promover el desarrollo de habilidades para comunicar, negociar y apoyar soluciones pacíficas en casos conflictivos.

Se observa que en Colombia, aun cuando desde la misma Constitución Política y la Ley General de Educación y sus reglamentos, se establece el marco legal que regula la convivencia escolar y muy a pesar de todos los esfuerzos que el estado colombiano realiza para mejorar su educación y llevarla a los mejores cánones de calidad, es un país donde existe una amenaza latente referida a la historia de violencia vivida.

En consecuencia, la educación en Colombia, se ve sometida a las lógicas del conflicto armado, éste ha generado, entre otros fenómenos, el riesgo de perder la vida de estudiantes y docentes, la vulneración del derecho a la educación de niños y jóvenes, el derecho al trabajo digno y seguro de los docentes, la ocupación temporal de las instalaciones escolares y el uso de la escuela como cuartel que, en ocasiones, tiene como consecuencia directa la suspensión de actividades en la escuela y en otros casos, el desplazamiento, emplazamiento, amenazas, para el personal (docentes y estudiantes) y líderes sociales de las diferentes comunidades, tanto en el área urbana como rural.

Según Peña (2018), cada doce (12) días asesinan un docente en Colombia. La inseguridad ha afectado todo proceso curricular y extracurricular en Colombia. El tema del impacto del conflicto armado en las escuelas ha sido trabajado por las Naciones Unidas recientemente, a partir de la década del 90 . 
Destaca el informe de la experta Graça Machel, (1996 c.p. Romero 2011) quien ya advertía sobre los riesgos de las escuelas en contextos de conflicto armado:

... En tiempos de guerra las escuelas sirven de objetivos, lo cual se debe en parte a su gran visibilidad. En las zonas rurales, tal vez el edificio de la escuela sea la única estructura permanente sustancial y por ello es muy probable que reciba disparos o sea objeto de cierre o de saqueos. La agresión y violación de los Derechos humanos, se produce a través de ataques con explosivos, detonación de explosivos por control remoto y disparos en las entradas, los patios de juego y los despachos de las escuelas en actos especiales; los asesinatos selectivos, las desapariciones, los secuestros, el exilio forzoso, detención ilegal, la encarcelación, la tortura, las mutilaciones, las violaciones cometidas por los soldados y las fuerzas de seguridad a estudiantes y profesoras; el reclutamiento de niños soldados y la ocupación y destrucción de establecimientos educativos; además, la deserción escolar como efecto colateral de la situación irregular que se vive en la escuela Naciones Unidas (p. 58).

Lo antes mencionado hace apenas lógico que se dé la violencia escolar dado que el aula de clases es el mejor laboratorio, reflejo y expresión de las construcciones y lógicas sociales. Según los criterios de Ovejero (2019) el conflicto escolar está asociado al Bullying, una conducta violenta, un comportamiento intencional, que se repite en el tiempo, en donde se presenta una situación de poder y fuerza entre el agresor y su víctima siendo muy frecuente la exclusión y el rechazo social.

En este sentido, la Unesco (2007) expresa que Colombia es uno de los países que registra mayor número de atentados contra el espacio escolar; el conflicto y los actores armados tanto regulares como no oficiales, situación que ha propiciado el desplazamiento masivo de comunidades, con la consecuencia lógica del abandono de la escuela por parte de los niños y niñas, por cuanto los actores armados controlan la movilidad, obstaculizan el acceso a la escuela $\mathrm{y}$ en ocasiones, por los enfrentamientos la escuela se cierra temporal o definitivamente.

Resulta claro entonces, inferir que la escuela colombiana ha perdido su razón de ser, y corresponde, por tanto, mediante estrategias de formación para la paz, donde el docente juega un papel estelar, establecer entre todos los actores del hecho educativo la sana convivencia, sin distingos de ninguna naturaleza.

En este sentido, la convivencia no se ha de pensar sólo como la ausencia de violencia, sino que ha de entenderse como el establecimiento de relaciones interpersonales y grupales satisfactorias que favorecen un clima de confianza, respeto y apoyo mutuo en la institución escolar, que potencia, asimismo, el funcionamiento democrático de la escuela, lo que, a su vez, favorece la existencia de relaciones positivas entre los miembros de la comunidad escolar.

En efecto, un informe de la Organización de las Naciones Unidas para la Educación, la Ciencia y la Cultura -Unesco- (2008), expresa que para disfrutar de una convivencia positiva ésta debe estar anclada en los siguientes pilares: Aprender a conocer, aprender hacer, aprende a vivir juntos, aprender a ser.

Efectivamente, estos pilares para la convivencia se sustentan en los principios de Delors (1996 c.p. Garretón 2013), los mismos enfatizan la importancia de conocerse a sí mismo, desarrollarse integralmente como persona, y fortalecer la responsabilidad en el destino propio y en el colectivo, lo que implica 
jerarquizar, ordenar, sistematizar el saber, adquirir medios para la comprensión, tener una base de conocimientos y al mismo tiempo aprender a aprender y a desaprender. Es el desarrollo de todas las potencialidades del individuo para poder enfrentar los desafíos que plantea la vida y al mismo tiempo es un llamado a aceptar y reconocer la interdependencia económica y cultural, lo que requiere educación en la tolerancia, el respeto, la solidaridad, así como desarrollar la inteligencia interpersonal.

Estos principios invitan a conocer mejor a los demás, su historia, tradiciones, espiritualidad, entre otros; hacerlo es aceptar la diversidad y los valores compartidos en una sociedad democrática. Indudablemente, la escuela como bastión de la cultura de los grupos sociales es la llamada a crear, mediante su promoción en el ámbito escolar, espacios de convivencia donde la diversidad tenga cabida de forma natural y espontánea.

Las personas necesitan la convivencia como capacidad innata para vivir con sus pares, dentro de un marco de respeto mutuo y muestras de solidaridad; la convivencia se da en la interrelación entre los diferentes miembros de una comunidad, e incide significativamente en el desarrollo ético, socio-afectivo e intelectual de los integrantes del grupo social; enfatizándose ésta en la comunicación, empatía, autocontrol, asertividad e inteligencia emocional que se ponga de manifiesto.

Según Ortega y Del Rey (2004 c.p. Garretón 2013) la convivencia escolar es una significación, sustentada en el proceso de enseñanza aprendizaje, que sugiere procesos que responden al bien común y la vida en democracia de un centro escolar. De allí que no pueda concebirse la convivencia sin la consabida aceptación de la diversidad. Su importancia radica en la atención e intervención pertinente y oportuna tras la aparición de episodios violentos o dramáticos, o sea las disrupciones o dificultades para convivir que se presentan y afectan la convivencia interna y externa de la comunidad educativa.

Según Palomino y otros (2010, p. 89), "la convivencia escolar se fundamenta, en los siguientes principios normativos: -principio de subordinación, -igualdad y no discriminación, -legalidad, Información, formación". Este autor resalta el principio de subordinación, por estar éste sujeto a la ley colombiana y a las normas internacionales de protección humana ratificadas por el estado. De igual manera, considera que acatar el principio de igualdad y no discriminación, elimina toda forma de arbitrariedad. Se valida la exclusión de toda forma de atropello; puede parecer una utopía, pero también sería el inicio de la equidad como condición de paz para la convivencia.

Se percibe en este planteamiento que la convivencia escolar tiene como objetivo central en las instituciones educativas establecer normas y códigos de interacción formal e informal para vivir armónicamente o hacer frente a los diversos conflictos de la vida humana. (Palomino y otros 2010).

Oros de los principios que fundamentan la convivencia escolar son: el principio de legalidad, para que al describir los comportamientos que deban corregirse, las sanciones sean proporcionales a las faltas. Este principio actualmente es válido y se maneja en variados ámbitos y contextos. El principio de información, porque las normas deben ser conocidas por todos los miembros de la comunidad educativa, y finalmente el principio de formación ya que las normas de convivencia deben tener un carácter formativo.

En el mundo dinámico, de cambios vertiginosos y cada vez más diversos, es un requerimiento básico aceptar y respetar al "otro" con sus singularidades personales y culturales; no es meramente "tolerar", es un 
desafío el aceptar y respetar en todas sus diferencias y enriquecerse de esta manera en la convivencia.

Los referidos principios hacen un llamado de atención al desarrollo de conductas prosociales para el mantenimiento de la convivencia y brindan la oportunidad de ser considerados guías en el manejo justo y equitativo de la diversidad étnica colombiana en pro de comportamientos que conduzcan a la armonía en las escuelas.

En este sentido, es necesario entender que los afrodescendientes en Colombia conforman un grupo significativo y heterogéneo, cohesionados e identificados no solo por su color de piel, el carácter más intrínseco es la estructuración de sus identidades diversas unidas por la lucha denodada, por la defensa de sus derechos a la no discriminación racial, la xenofobia $\mathrm{y}$ formas de intolerancia; la desigualdad, marginación y estigmatización social, el esfuerzo por lograr la participación y representación política y social, el acceso justo y equitativo a la educación, los servicios de atención de salud y la igualdad de acceso en el mercado laboral, el reconocimiento y valoración de la diversidad étnica y cultural de los afrodescendientes y la aceptación de las religiones de origen africano, entre otros aspectos.

Es así como, la propuesta de la Organización Mundial de la Salud (OMS, 1993 c.p. Martínez, 2014) de las 10 Habilidades para la Vida, logra reconocimiento por aplicabilidad universal y congruencia con los principios de Delors desde 1996. El ideal de la formación del ser humano es asumirse como un ser de dimensión holística, capaz de vivir con satisfacción de sí mismo en relación armoniosa con el medio ambiente y como miembro activo y responsable de la comunidad mundial.

En este mismo orden de ideas, Jares, (2006 c.p. Conde 2012) resalta que la convivencia pone de manifiesto diferentes estilos de conductas que han sido de alguna manera influenciadas por diversos factores tales como: a) el sistema económico social; b) el menoscabo y el respecto de los valores básicos de convivencia; c) la mayor complejidad y heterogeneidad social; d) la pérdida del liderazgo educativo de los dos sistemas tradicionales de educación; la familia y el sistema educativo; e) la mayor aparición y visibilidad de la violencia.

Expresa el autor antes mencionado que la convivencia es influenciada por el sistema económico social, sobre todo cuando éste se fundamenta en ganar a cualquier precio, el interés económico tiene el primer lugar y la consideración de los seres humanos como recursos o medios y no como fines; el autor reconoce que mucho de esta condición está presente en el sistema económico social colombiano, lo que incide en el tipo de convivencia escolar que se manifiesta en las instituciones educativas.

De igual forma considera el autor que el menoscabo al respecto de los valores básicos de convivencia, o sea los siempre llamados, educación básica o urbanidad, trae como consecuencia, específicamente en Colombia, el tipo de sociedad presente, la cual es cada vez más individualista, consumista, deshumanizada, entre otras características; y los cambios culturales en las relaciones sociales que originan mayor complejidad y heterogeneidad social, debido a la globalización y al fenómeno de pasar de ser un país exportador de emigrantes a ser receptor de éstos.

Igualmente, la pérdida del liderazgo educativo en los sistemas tradicionales de educación: la familia y el sistema educativo, que en buena medida se explican por los cambios sociales, la transformación del papel de ambos sistemas, el empuje y protagonismos 
paulatino de los medios de comunicación, adopción de comportamientos sociales de determinadas culturas grupales, el desconcierto $\mathrm{y}$ un claro descompromiso en sectores significativos de las familias y el profesorado, así como la aparición y visibilidad de la violencia desde muy temprana edad como signo de identidad; es considerado por Jares (2006 c.p. Conde. 2012) como un factor de influencia sobre la convivencia.

Finalmente, el mencionado autor considera que los grados de prevención individual, relacional y comunitario que se ejerzan es otro factor relacionado con el estilo de convivencia que se pone de manifiesto. Resalta que el grado de prevención individual centra su atención en las características del individuo y pretende identificar los factores biológicos de la historia personal que influyen en el comportamiento de una persona, como impulsividad, bajo nivel educativo, abuso de sustancias psicotrópicas y antecedentes de comportamiento agresivo o de haber sufrido maltrato, entre otros, lo que aumenta la probabilidad de ser víctima o responsable de actos violentos.

En cuanto al nivel de prevención relacional, éste indaga el modo en el que las relaciones sociales cercanas aumentan el riesgo de convertir a una persona en víctima o responsable de actos violentos; los compañeros, la pareja y los miembros de la familia tienen el potencial de configurar a un individuo a través de un amplio abanico de experiencias. En esta línea, Martínez-Otero (2001, c.p. Uribe, 2015, p. 39) menciona que "los problemas familiares tienen gran impacto en el desarrollo de los niños".

En relación al nivel de prevención comunitario, explica Jares, (2006 c. p. Conde 2012) que éste examina los contextos de la comunidad como la escuela, el lugar de trabajo y el vecindario, para identificar las características de estos ámbitos que se asocian con ser víctimas o agresores. Las investigaciones sobre la violencia muestran que determinados ámbitos comunitarios favorecen la violencia más que otros, entre ellos, las zonas de pobreza o deterioro físico, o donde hay poco apoyo institucional.

En efecto, son muchos los factores que obstaculizan la convivencia escolar armónica, éstos pueden clasificarse desde conductas disruptivas e indisciplina, problemas relacionales entre el estudiantado, falta de Implicación familiar, desmotivación y desinterés académico, medidas educativas inadecuadas, ausentismo, así como el hecho de ser percibido diferente.

Es así como los problemas de convivencia de cualquier lugar del mundo, son los mismos que se presentan en Colombia en los diferentes contextos y muy especialmente en el contexto escolar, entre los que se pueden mencionar: agresividad (física y psicológica) entre pares, agresión verbal, indisciplina, presencia de objetos que interfieren en el aula escolar (celulares, maquillaje, ruido), tramitación inadecuada del conflicto, comportamientos antisociales, fallas en el acatamiento de normas de disciplina, poca concentración de los estudiantes en las aulas escolares, diferencias culturales, riñas y alegatos entre estudiantes, violencia callejera, violencia intrafamiliar, de marginalidad o por desplazamiento a causa de conflictos armados como es el caso específico de Colombia.

Los problemas antes mencionados son dificultades que se encuentran presentes en Colombia por más de 50 años, Bethencour (2001 c.p. Palomino 2010) y que deben ser abordados desde aprendizajes cooperativos para el desarrollo de habilidades y de competencias personales y grupales; así como controlar los factores influyentes en su aparición, a partir del conocimiento de las relaciones intrafamiliares, los grupos de pares y 
las amistades con los cuales los estudiantes mantienen contacto en sus horas extraescolares, entre otros, por ser el escenario escolar el reflejo de lo que sucede en la familia, la comunidad y por ende en la sociedad en general.

La escuela refleja la realidad que viven los pueblos, sus aulas muestran el clima familiar de sus estudiantes, de sus familias y el clima de los diferentes contextos sociales. Sabiamente, considera Barroso (2009) que, si la crisis que se enfrenta es de valores, la solución también debe venir dada en la siembra de valores, lo que es posible en la familia y en la escuela por ser estos dos contextos los mejores y más significativos laboratorios sociales; para trabajar en la solución.

El principio de la escuela jamás ha sido ser una isla, es lógico, pues, que, en un país marcado por la violencia, la escuela sea el reflejo de su realidad social e histórica, consecuencialmente, es de la escuela el gran desafío de crear espacios de convivencia donde conductas como: reforzar el sentido de identidad nacional y pertenencia, la detección de alertas tempranas y la intervención pertinente mediante estrategias pedagógicas, el aprestamiento escolar, el establecimiento y manejo de canales de comunicación asertiva, la formación en valores, principios y comportamientos éticos, e implementación de espacios pedagógicos pertinentes al acto educativo, aseguren la formación de estudiantes idóneos.

Idoneidad que debe ponerse de manifiesto no solo en el manejo de conocimientos científicos y tecnológicos, sino sobre todo en la capacidad desarrollada por los estudiantes para convivir en armonía, asumir comportamientos éticos que faciliten su crecimiento en el respeto por la diferencia, interactuar con responsabilidad social y fortalecer su sentido de resiliencia para superar las dificultades en armonía con los cánones de la prosocialidad y alineados a comportamientos potencialmente derivados de un autoestima adecuado, que permite creer en sí mismo, pero también en el otro, para lo cual es necesario el manejo efectivo y eficiente de los elementos que conforman la convivencia como son: la comunicación, el apoyo mutuo, el respeto, la organización, la cordialidad, el sentido de pertenencia, y la participación efectiva.

\section{CONCLUSIONES}

Aunque en Colombia, frente a la problemática de la convivencia y la necesidad de incentivar comportamientos prosociales, se han tomado variadas iniciativas en el ámbito educativo, tales como el trabajo pedagógico, las aulas de paz, la construcción de los estándares de competencias ciudadanas, los talleres de socialización donde se fijaron estándares de convivencia y el Plan de Desarrollo 2014-2018, con el objetivo de que los estudiantes asistan al colegio durante gran parte del día para garantizar una mayor exposición a un ambiente formativo supervisado, se hace necesario revisar los logros de estas iniciativas, por cuanto en algunos sectores educativos persisten los problemas de convivencia y violencia dentro de los ambientes educativos.

Todas y cada uno de las iniciativas antes mencionadas persiguen, desde la educación, dar respuesta a la problemática personal y social de la educación colombiana con el objetivo de empoderar a los estudiantes con herramientas de asertividad, solidaridad y respeto a utilizar en sus relaciones tanto en el ámbito educativo, como en el contexto social, para alcanzar así una convivencia escolar efectiva que rinda frutos que impacten su desenvolvimiento y rendimiento así como la armonía de las relaciones de la comunidad educativa.

Consecuentemente, se hace importante reconocer la escuela como un espacio de 
creación y construcción de conductas prosociales que logren superar los comportamientos de violencia y racismo que caracterizan a la sociedad colombiana, lo cual significa reconocerse como personas con igualdad de derechos, a fin de cumplir con el sueño de una sociedad donde la escuela a través de la educación fortalezca la experiencia de participación con respeto a los matices culturales de cada comunidad y donde lo aprendido conforme la base para una convivencia efectiva con espacios para el diálogo y el entendimiento.

\section{REFERENCIAS}

Barroso, M. (2009). Ser Familia. Venezuela. Editorial Galac, S.A.

Conde, V. (2012) Estudio de la gestión de la convivencia escolar en centros de Educación Secundaria de Andalucía: una propuesta de evaluación basada en el Modelo EFQM. (Tesis doctoral) Universidad de Huelva. Departamento de Educación. Recuperado de: http://rabida.uhu.es/dspace/bitstream/ha ndle/10272/6438/Estudio_de_la_gestion_ de_la_convivencia.pdf?sequence $=2$

Delors, J. (1996). Los cuatro pilares de la educación, en La educación encierran un tesoro. Informe a la UNESCO de la Comisión internacional sobre la educación para el siglo XXI. Madrid: Santillana/UNESCO

El Decenio de las Naciones Unidas de la Educación para el Desarrollo Sostenible EDS-(2005-2014).

Foro Internacional sobre Prevención y Manejo de la Intimidación Escolar (2007). Bogotá. Colombia.

Garretón-Valdivia, P. (2013). Estado de la convivencia escolar, conflictividad y su forma de abordarla en establecimientos educacionales de alta vulnerabilidad social de la provincia Concepción: Chile.

Hurtado, J. (2010) Metodología de la Investigación. Guía para la comprensión holística de la ciencia. Ediciones Quirón. Caracas, Venezuela.

Martínez, V. (2014). Habilidades para la vida: Una propuesta de formación humana. Itinerario Educativo, xxviii (63), 61-89. file:///C:/Users/\%20/Downloads/1488Texto\%20del\%20art\%C3\%ADculo-31881-10-20150625\%20(2).pdf

Ministerio de Educación Nacional (MEN). (2017) Plan Decenal de Educación 20172026. El camino hacia la calidad y la equidad

http://www.plandecenal.edu.co/cms/med ia/herramientas/PNDE\%20FINAL_ISBN\% 20web.pdf

Ministerio de Educación Nacional (MEN). (2013) Ley de Convivencia Escolar, Ley 1620 de marzo 15 de 2013, al tenor de la Ley General de Educación, Ley 115 de 1994 y en Marco de la Constitución Política de Colombia. Presidente de la República, Congreso de Colombia.

Ministerio de Educación Nacional (s.f). Lineamientos Curriculares. https://www.mineducacion.gov.co/1759/ w3-article-339975.html?_noredirect=1

Mockus, A. (2002). Guías pedagógicas para la convivencia escolar. Ley 1620 de 2013 y Decreto 1965 de 2013. Recuperado de: https://redes.colombiaaprende.edu.co/ntg /men/pdf/Guia\%20No.\%2049.pdf

Ovejero, A. (2019). Fracaso escolar y reproducción social. La cara oscura de la escuela. ISBN: 978-84-09-09531-5 Fecha de edición: marzo 2019 Diseño de portada: Gonzalo Ovejero Maquetación: Gonzalo Ovejero Contacto: tasio@psi.uva.es Libro descargable desde anastasio.ovejero.net. Barcelona (c) 2019 Creative Commons.

Palomino, M. y Dagua, P. (2010). Los problemas de convivencia escolar: percepciones, factores y abordajes en el aula. Revista Suplemento Memorias V Encuentro Volumen 9, Número 2 Recuperado de: https://academia.unad.edu.co/images/inves tigacion/hemeroteca/revistainvestigaciones /Volumen9numero2_2010/8.\%20LOS\%20P ROBLEMAS\%20DE\%20CONVIVENCIA.pdf 
Pérez, R (2007). Guía pedagógica manual de convivencia. Recuperado de: https://esstatic.z-

dn.net/files/d97/9e19348e1313b71cbed 018406feb7636.pdf

Romero, M. (2011). Unesco, La educación víctima de la violencia armada, Paris: UNESCO, 2007, [Consultado el 7 de febrero de 2019], disponible en línea en www.unicef.org/informes

UNESCO, U. (2015). Banco Mundial. Declaración de Incheon. Educación, 2030

Unesco (2008). "La educación inclusiva: el camino hacia el futuro" conferencia internacional de educación Cuadragésima octava reunión Centro Internacional de Conferencias, Ginebra 25 a 28 de noviembre 2008 http://www.ibe.unesco.org/fileadmin/us er_upload/Policy_Dialogue/48th_ICE/CON FINTED_48_Inf_2_Spanish.pdf
Unesco (2007). La educación víctima de la violencia armada .Recuperado de: https://unesdoc.unesco.org/ark:/48223/ pf0000187443_spa

Uribe, N. (2015). La convivencia escolar desde la perspectiva de la resiliencia: Un apoyo a la gestión educativa. (Tesis). Universidad Libre de Colombia. https://repository.unilibre.edu.co/bitstre am/handle/10901/7864/UribeCorderoN ancyEdith2015.pdf

Vélez, C. (2012). Estudio de la gestión de la convivencia escolar en

centros de Educación Secundaria de Andalucía: una propuesta de evaluación basada en la excelencia-EFQ- 